\title{
ORIGINAL ARTICLE \\ Relative validity of a short food frequency questionnaire assessing adherence to the Norwegian dietary guidelines among colorectal cancer patients
}

\author{
Hege Berg Henriksen', Monica Hauger Carlsen', Ingvild Paur', Sveinung Berntsen², \\ Siv Kjølsrud Bøhn', Anne Juul Skjetne', Ane Sørlie Kværner', Christine Henriksen', \\ Lene Frost Andersen', Sigbjørn Smeland ${ }^{3,4}$, Rune Blomhoff',5*
}

\begin{abstract}
'Department of Nutrition, Institute of Basic Medical Sciences, University of Oslo, Oslo, Norway; ${ }^{2}$ Faculty of Health and Sports Science, University of Agder, Kristiansand, Norway; ${ }^{3}$ nstitute of Clinical Medicine, University of Oslo, Oslo, Norway; ${ }^{4}$ Division of Cancer Medicine, Oslo University Hospital, Oslo, Norway; ${ }^{5}$ Department of Clinical Service, Division of Cancer Medicine, Oslo University Hospital, Oslo, Norway
\end{abstract}

\section{Abstract}

Background: The Norwegian food-based dietary guidelines (FBDG) aim at reducing the risk of developing chronic diseases and promote overall health. We studied the effect of the Norwegian FBDG in colorectal cancer (CRC) patients. There is a need for a time-efficient dietary assessment tool measuring adherence to these guidelines in patients treated for dietary dependent cancer, such as CRC patients.

Objective: To evaluate a new short food frequency questionnaire (NORDIET-FFQ), developed to estimate adherence to the Norwegian FBDG among CRC patients.

Design: Eighty-one CRC patients from both study groups in the Norwegian Dietary Guidelines and Colorectal Cancer Survival study, an ongoing dietary intervention, completed both the short 63-item NORDIET-FFQ and a 7-day weighed food record.

Results: The NORDIET-FFQ was on group level able to estimate intakes of fruits, vegetables, unsalted nuts, fish, fatty fish, high fat dairy products, unprocessed meat, processed meat, red meat, water, sugar-rich beverages, alcoholic drinks, and sugar- and fat-rich foods. Ranking of individuals according to intake was good $(r=0.31-0.74)$ for fruits and vegetables, fruits, unsalted nuts, whole grain products, sugar-rich cereals, fish, fatty fish, dairy products, red meat, water, sugar-rich beverages, alcoholic beverages, and sugar- and fat-rich foods. The NORDIET-FFQ was able to identify the individuals who did not fulfil the recommendations of fruits, vegetables, unsalted nuts, whole grains, low-fat dairy products, processed meat, water, alcoholic beverages, and sugar- and fat-rich foods (sensitivity: 67-93\%).

Conclusions: The NORDIET-FFQ showed good ability in to estimate intakes of plant-based foods, fish, dairy products, meat, and energy-dense foods; adequate ranking of individuals according to intake of most recommendations except for unprocessed meat, processed meat, and vegetables; and importantly a good ability to identify those patients in need of dietary counselling for foods that are known to modulate the risk of CRC. Trial registration: National Institutes of Health ClinicalTrials.gov; Identifier: NCT01570010.

Keywords: dietary assessment tool; weighed food records; validity; dietary intake; food based dietary guidelines; cancer

To access the supplementary material, please visit the article landing page

Received: 2 March 2017; Revised: 3 January 20 8; Accepted: 24 January 20 I 8; Published: 22 February 2018

Most countries develop national food-based dietary guidelines (FBDG) (1-4). In 2011, the health authorities in Norway published updated FBDG, encouraging intake of a plant based diet with ample amounts of vegetables, fruits, berries, whole grains, and fish and limited amounts of red and processed meat, salt, sugar, alcohol, and high-energy foods. The Norwegian FBDG are similar to the national FBDG in most other developed countries $(3,5)$. A major aim of the Norwegian FBDG is to reduce risk of lifestyle related diseases such as cancer, cardiovascular diseases, diabetes, and obesity. 
Colorectal cancer (CRC) is the third most common cancer in Norway and second most common cause of cancer death (6). Low intake of whole grains, foods containing dietary fibre, dairy products and high intake of red and processed meat, alcoholic drinks as well as increased body fat have been associated with higher risk of developing CRC (4). All of these risk factors are included in the Norwegian FBDG, but the national guidelines have a much broader perspective than only these risk factors related to CRC.

Little is known about the effect of diet on disease outcomes and survival in CRC patients. We have therefore initiated a large, long-term randomised controlled trial (RCT) (CRC-NORDIET) to study the effect of diet in CRC patients post-surgery (7). Instead of only focusing on the dietary factors associated with risk of CRC, participants in the CRC-NORDIET study are instructed to follow a dietary pattern that is consistent with the Norwegian FBDG, since CRC patients have increased risk of lifestyle-related co- and multimorbidities.

It is therefore of interest to assess to what extent CRC patients in the CRC-NORDIET study comply with the Norwegian FBDG, both those foods that are causally related to CRC as well as those foods that are related to other lifestyle-related co-and multimorbidities.

Dietary intervention studies as well as nutrition education and counselling would benefit from a user friendly, short dietary assessment tool. In nutritional research, a variety of comprehensive dietary assessment tools are used, including food frequency questionnaires (FFQs), 24-h dietary recall, and food records (8). The FFQ is an established assessment method often used when investigating the effects of diet on disease outcomes in populations or groups of dietary interventions $(9,10)$. Since most FFQs aim at capturing total habitual diet and therefore often include 200-300 questions, they are time-consuming for the respondents to complete, and data handling may be complex for the researcher $(8,11-17)$. Short FFQs are less time-consuming for both the patient and the researcher. Short FFQs designed to cover a recent time period (i.e. 1-2 months) have been shown to be useful for identifying dietary changes in individuals and in intervention studies $(13,17-20)$ and may also be applicable to dietary counselling of patients in a clinical setting (11).

In recent years, a number of short FFQs have been developed to monitor adherence to food recommendations (11,21-27); however, none of these assess adherence to a national FBDG. As part of the ongoing CRC-NORDIET study (7), we developed a short semi-quantitative FFQ (NORDIET-FFQ), designed to estimate the adherence to the Norwegian FBDG. The objective of the present study was to validate the ability of the NORDIET-FFQ to assess adherence to the Norwegian FBDG in CRC patients.

\section{Methods}

\section{Subjects and study design}

Men and women aged 50-80 years old, with non-metastatic CRC (International classification of diseases (ICD)-10 18-20), staged I-III according to the TNM (tumour node metastases) staging system (28), and participating in the CRC-NORDIET study (7) were invited to take part in the present validation study. The participants in the validation study were recruited from both intervention $(n=48)$ and control groups $(n=33)$ at the follow-up visit 6 months after baseline of the study, from January 2014 to October 2015. About $15 \%$ of the participants received chemotherapy post-surgery, of which the mean time from last chemotherapy injection to the validation study start was 155 days. Hence, none of the participants included in the validation study underwent adjuvant treatment during the time frame covered by the dietary assessment tools. All seasons during a year were included. The participants completed the self-administered NORDIET-FFQ at the study centre and received a digital scale and weighed record (WR), to be completed at home within 2 weeks (Fig. 1).

\section{Ethics and approvals}

The CRC-NORDIET study is being carried out in accordance with the Helsinki Declaration and informed consent was obtained from all participants. The study was approved by the Regional Committees for Medical and Health Research Ethics (REC Protocol Approval 2011/836) and by the data protection officials at Oslo University Hospital, Oslo, Norway and Akershus University Hospital, Lørenskog, Norway. The study is registered on the National Institutes of Health ClinicalTrials.gov (Identifier: NCT01570010).

\section{Characteristics of the participants}

Anthropometric measurements (weight, height, and hipand waist circumference) were measured as previously described (7). Level of education, smoking status, and tumour location were retrieved from other questionnaires within the clinical trial and from medical records.

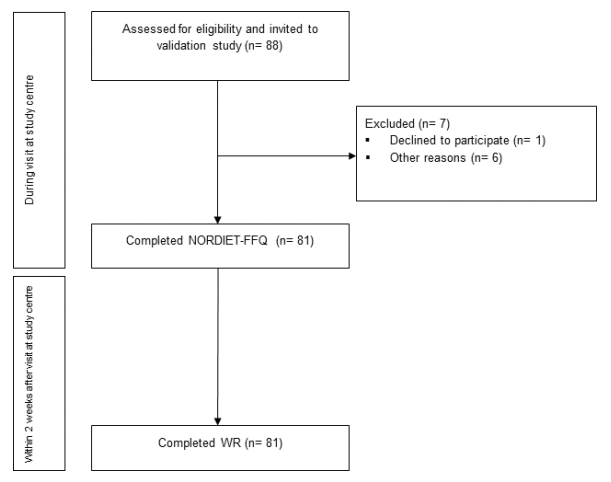

Fig. 1. Study design and timeline of the validation study. 


\section{NORDIET-FFQ}

The NORDIET-FFQ is a short semi-quantitative 63-item FFQ designed to assess dietary intake (in grams per day) over the previous $1-2$ months. It takes on average $15 \mathrm{~min}$ to complete. The questions in the NORDIET-FFQ correspond to the food groups relevant for the Norwegian FBDG (3). The NORDIET-FFQ is not designed for estimation of total energy or nutrient intake.

The NORDIET-FFQ included questions of both frequency (how often the food item was consumed) and amount of the food items. The 63 questions cover the following food groups: fruit, berries, nuts, vegetables, cereals, beverages, cakes, sweet candy, breads and spreads, oils, margarine and butter, dairy products, fish, meat, rice, pasta, and dietary supplements. The questionnaires were checked for completeness by the researchers, so that incomplete answers could be corrected. Data from the NORDIET-FFQ were scanned and the image files transformed into data files using Cardiff Teleform 2006 Software (6.0) (Datascan, Oslo, Norway). The software proofread the answers in the NORDIET-FFQ pending approval by the handler. When values were missing the following rules were used: (1) when frequency was reported but amount was missing, the lowest amount was registered; (2) when amount was reported but the frequency was missing, the lowest frequency above 0 was registered; (3) when both frequency and amount were missing, they remained as missing values; (4) if the amount of food was reported and the frequency was reported as zero, the amount was removed.

The food composition database and nutrient calculation system developed at the Department of Nutrition, University of Oslo (KBS, version 4.7, 2010, AE-10), was used for the calculations of food and beverage intake (29).

\section{Seven-day weighed food record}

All participants were provided with a WR and a digital scale (5 kg kitchen scale, Clas Ohlson Model CFC2025, Oslo, Norway) and were instructed how to weigh and record all foods and beverages consumed during a period of 7 consecutive days. The participants returned the completed WRs to the study centre at Department of Nutrition, University of Oslo, by postal mail. Dietary data were retrieved from the WR and manually coded and imported into the food database AE-10 and KBS software system (KBS, 2010). The manual coding and import of data were done by two researchers (HBH and SFØ), in accordance with a protocol developed at the Department of Nutrition, University of Oslo. All of the 81 WRs were included in the analyses.

\section{Dietary recommendations}

\section{Quantitative Norwegian FBDG}

The questions about food and beverage intake in the NORDIET-FFQ were grouped according to the quantitative recommendations of the Norwegian FBDG as defined in Table 1. The main recommendations are listed in Table 1 together with the quantitative limits required to fulfil the Norwegian FBDG. The last two columns of Table 1 list all the specific questions in the NORDIET-FFQ that are included in calculation of adherence to the recommendations. Whole grain intake was estimated from whole grain products using a whole grain factor $(30,31)$. The lower range of recommended fish intake was used in the definition of daily intake (i.e. $300 \mathrm{~g} /$ week or $43 \mathrm{~g} / \mathrm{d}$ ).

\section{The translation of qualitative Norwegian FBDG into quantitative limits}

In order to measure adherence to the qualitative recommendations of the Norwegian FBDG we had to translate these qualitative recommendations into quantitative values and lower limits of intake (Table 2). The quantitative values used in the present paper are listed in the footnotes of Table 2. The last two columns of Table 2 list all the specific questions in the NORDIET-FFQ that are included in calculating adherence to the recommendations. Qualitative recommendations that included the terms 'limit' or 'reduce' were quantitatively defined as the highest acceptable amount of daily intake. For example, the recommendation regarding processed meat reads, 'Limit the intake of processed meat'. In this case we a priori set the quantitative limit required to fulfil the Norwegian FBDG to $\leq 20$ $\mathrm{g} / \mathrm{d}$. This is equivalent to a fast-food meal or dinner with processed meat once a week. Moreover, the term 'preference' included in the recommendation of unprocessed meat (i.e. 'Preference should be given to the consumption of unprocessed meat') was defined as meaning that intake of unprocessed meat should exceed the intake of processed meat.

For recommendations where a daily intake of specific food items was specified, this was defined as the minimum amount that should be consumed daily with some modifications. For example, a moderate intake of unsalted nuts (about $140 \mathrm{~g} /$ week or $20 \mathrm{~g} / \mathrm{d}$ ) is recommended. However, because nuts are protein and energy dense, high intakes may lead to weight gain. Therefore, the recommendation of unsalted nuts was defined as a daily intake of at least 20 $\mathrm{g}$ or more among normal weight [body mass index (BMI) $<25$ ] individuals and between 20 and $30 \mathrm{~g} / \mathrm{d}$ among overweight and obese individuals (BMI $\geq 25$ ). In the case of dairy products, the recommendation states that 'Low-fat dairy products should be included in your daily diet'. The recommended daily amount was defined as at least half a portion of low-fat dairy products per day. Water is recommended as the primary choice of beverage; however, there is no quantitative daily recommendation of water intake in the Norwegian FBDG. Therefore, we determined that, to fulfil this recommendation, at least $25 \%$ of the daily beverage intake should be water. 
Table 1. The quantitative NFBDG and corresponding questions in the NORDIET-FFQ

Quantitative NFBDG
$\mathrm{I} . \mathrm{I}$ It is recommended to eat at least
five portions, corresponding to at
least $500 \mathrm{~g}$ altogether, of vegetables,
fruit and berries every day.

I.2 About half of this intake should be in the form of fruit and berries.

I.3 About half of this intake should be in the form of vegetables. ${ }^{a}$

2.I Eat at least four portions of whole grain products every day. Four portions of whole grain products corresponds to about 70-90 g of whole grains per day. ${ }^{c, d}$

2.2 At least half of the total consumption of grain products should be in the form of whole grains. ${ }^{c, d}$

Intake required to fulfil NFBDG

$\geq 500 \mathrm{~g} / \mathrm{d}$

$\geq 250 \mathrm{~g} / \mathrm{d}$

$\geq 250 \mathrm{~g} / \mathrm{d}$

Women: $\geq 70 \mathrm{~g} / \mathrm{d}$

Men: $\geq 90 \mathrm{~g} / \mathrm{d}$

Whole grain $(g / d)>50 \%$ of total grains (g/d)

$\geq 43 \mathrm{~g} / \mathrm{d}(300 \mathrm{~g} /$ week $)$

3.I Weekly consumption
$g$ of fish is recommended.

3.2 It is recommended that at least $200 \mathrm{~g}$ of the intake should be of fatty fish.

4.I Limit the consumption of red meat (beef, pork, lamb, and goat) to $500 \mathrm{~g} /$ week.
Foods and drinks included to calculate dietary intake $(\mathrm{g} / \mathrm{d})$

Large fruits (e.g. apple, nectarine, banana, orange)

Medium fruits (e.g. clementine, kiwifruit, plum)

Small fruits (e.g. grapes)

Berries (frozen or fresh strawberries, bilberries, raspberries, etc.)

Dried fruits (e.g. raisins, apricot, prunes, dried apples)

Fresh fruits and vegetables used as spread on bread ${ }^{b}$

Garlic

Onion and leek

Tomatoes

Tomato sauce

Mixed salad

Vegetables (e.g. carrots, broccoli, cauliflower)

Juice [ I glass of juice $(2 \mathrm{dL})$ counts as one portion of fruit $(=100 \mathrm{~g})$; intake $>$ I glass does not count]

Large fruits (e.g. a whole apple, nectarine, banana, orange)

Medium fruits (e.g. clementine, kiwifruit, plum)

Small fruits (e.g. grapes)

Berries (frozen or fresh strawberries, bilberries, raspberries, etc.)

Dried fruits (e.g. raisins, apricot, prunes, dried apples)

Fresh fruits used as spread on bread $(50 \% \text { of subquestion } 9 \mathrm{~g})^{\mathrm{b}}$

Juice [I glass juice $(2 \mathrm{dL})$ counts as I portion of fruit $(=100 \mathrm{~g})$; intake $>$ I glass does not count]

Vegetables (e.g. carrots, broccoli, cauliflower)

Fresh vegetables used as spread on bread (calculated as $50 \%$ of subquestion $9 \mathrm{~g}$ in the NORDIET-FFQ) ${ }^{\mathrm{b}}$

Tomatoes

Tomato sauce

Mixed salad

Garlic

Onion and leek

Bread with $25-50 \%$ wholemeal flour (60\% cereals)

Bread with $50-75 \%$ wholemeal flour (60\% cereals)

Bread with $75-100 \%$ wholemeal flour (60\% cereals)

Wholemeal crisp bread

Sweetened cereals (e.g. Corn Flakes)

Unsweetened cereals (e.g. oatmeal porridge)

Brown riceWhole grain pasta

Bread with 0-25\% wholemeal flour (60\% cereals)

Bread with $25-50 \%$ wholemeal flour ( $60 \%$ cereals)

Bread with $50-75 \%$ wholemeal flour (60\% cereals)

Bread with $75-100 \%$ wholemeal flour (60\% cereals)

White crisp bread

Wholemeal crisp bread

Sweetened cereals (e.g. Corn Flakes)

Unsweetened cereals (e.g. oatmeal porridge)

White rice

Brown rice

White pasta

Whole grain pasta

Cakes, buns, waffles, sweet biscuits

Fatty fish (e.g. salmon, trout, herring, halibut)

Lean fish (e.g. cod, pollock, angler)

Processed fish (e.g. fish gratin, fish cakes) ( $40 \%$ fish)

Fish as spread (e.g. mackerel, smoked salmon, herring)

Fatty fish (e.g. salmon, trout, herring, halibut)

Fish as spread (e.g. mackerel, smoked salmon, herring)

Unprocessed red meat

Processed red meat

Red meat as spread 
Table 1. Continued

\begin{tabular}{|c|c|c|}
\hline Quantitative NFBDG & Intake required to fulfil NFBDG & Foods and drinks included to calculate dietary intake (g/d) \\
\hline $\begin{array}{l}\text { 5.I Cooking oils and margarine with } \\
\text { a low content of saturated fatty acids } \\
\text { and a high content of unsaturated } \\
\text { fatty acids should be used in prefer- } \\
\text { ence to similar products with a great } \\
\text { proportion of saturated fatty acids. }\end{array}$ & $\begin{array}{l}\text { Users of cooking oil, liquid marga- } \\
\text { rine, or soft margarine and non- } \\
\text { users of butter with high content of } \\
\text { saturated fatty acids }\end{array}$ & $\begin{array}{l}\text { Margarine, butter, and oil as spread } \\
\text { Margarine, butter, and oil in cooking }\end{array}$ \\
\hline $\begin{array}{l}\text { 6.I Consumption of alcohol is not } \\
\text { recommended. }\end{array}$ & $0 \mathrm{~g} / \mathrm{d}$ & $\begin{array}{l}\text { Beer with alcohol } \\
\text { Wine with alcohol } \\
\text { Liquor }\end{array}$ \\
\hline
\end{tabular}

NFBDG, Norwegian food-based dietary guidelines; NORDIET-FFQ, NORDIET food frequency questionnaire

${ }^{a}$ Not including legumes or potatoes.

bam not included.

'Not including sausage rolls, tortillas, hamburger bread, pizza dough, etc.

Whole grain factor used in calculation of intake of whole grains from whole grain products (bread contains $60 \%$ flour):

- Bread with $0-25 \%$ wholemeal flour: $(60 * 0) / 10,000=0$

- Bread with $25-50 \%$ wholemeal flour: $(60 * 25) / 10,000=0.15$

- Bread with $50-75 \%$ wholemeal flour: $(60 * 50) / 10,000=0.30$

- Bread with $75-100 \%$ wholemeal flour: $(60 * 75) / 10,000=0.45$

- Crisp bread =0

- Whole grain crisp bread $=1$

- Sweetened cereals $=0.25$

- Unsweetened cereals $=0.75$

Boiled rice and pasta contain $70 \%$ water and $30 \%$ cereal. Whole grain factor used in calculation of whole grain intake from rice and pasta:

- Brown rice $=0.30$

- White rice $=0$

- Whole grain pasta $=0.30$

- White pasta $=0$

e Not including shellfish, mussels, or roe.

Table 2. The qualitative NFBDG defined as quantitative recommendations and corresponding questions in the NORDIET-FFQ

Qualitative NFBDG

It is recommended to consume a moderate amount of unsalted nuts (about $140 \mathrm{~g} /$ week). ${ }^{\mathrm{a}}$

2.I Reduce cereals with high content of fat and sugar. ${ }^{d}$

3.I Low-fat dairy products should be included $\geq 100 \mathrm{~g} / \mathrm{d}^{\text {h }}$ in your daily diet. dff.g $^{\circ}$

3.2 The consumption of dairy products that contain high levels of saturated fat and/or a high energy content should be limited. $., j, k$

4.I Moderate consumption of unprocessed $\geq 20 \mathrm{~g} / \mathrm{d}^{\mathrm{m}}$ meat can be included in the diet

4.2 Preference should be given to the consumption of unprocessed meat.
Intake required to fulfil the dietary recommendations.(See footnotes for estimation of quantitative limits when the recommendations are not explicit.)

$\geq 20 \mathrm{~g} / \mathrm{d}$ nuts and $\mathrm{BMI}<25^{\mathrm{b}}$

$20 \mathrm{~g} / \mathrm{d} \leq$ nuts $<30 \mathrm{~g} / \mathrm{d}$ and $\mathrm{BMI} \geq 25^{\mathrm{c}}$

$<20 \mathrm{~g} / \mathrm{d}^{\mathrm{e}}$

$<20 \mathrm{~g} / \mathrm{d}^{\prime}$

Unprocessed meat $(\mathrm{g} / \mathrm{d})>50 \%$ of total meat (g/d)
Foods and drinks included to calculate dietary intake $(g / d)$

Unsalted nuts (e.g. almonds, peanuts, walnuts)

Sweetened cereals (e.g. Corn Flakes)

Cakes, buns, waffles, sweet biscuits

Low-fat dairy products

Reduced-fat cheese

lean milk

High-fat dairy products

High-fat cheese

Whole milk

Unprocessed red meat

Unprocessed white meat

Unprocessed red meat

Processed red meat

Unprocessed white meat

Processed white meat

Red meat as spread

White meat as spread 
Table 2. Continued

Qualitative NFBDG choice of drink.
Intake required to fulfil the dietary recommendations.(See footnotes for estimation of quantitative limits when the recommendations are not explicit.)

4.3 Limit the intake of processed meat.
Foods and drinks included to calculate dietary intake $(g / d)$

\section{$\leq 20 \mathrm{~g} / \mathrm{d}^{\mathrm{n}}$}

Water $(\mathrm{g} / \mathrm{d}) \geq 25 \%$ of total drinks (g/d)
5.2The consumption of drinks with added sugar, such as carbonated drinks, should be limited.

6.I Reduce intake of foods with high content of sugar and fat.

\author{
Processed red meat \\ Processed white meat \\ Red meat as spread \\ White meat as spread \\ Water (e.g. tap or bottled water) \\ Beer with alcohol \\ Wine with alcohol \\ Liquor \\ Beverages without added sugar (e.g. mineral \\ water, light soft drinks) \\ Juice (e.g. apple juice, orange juice, etc.) \\ Beverages with added sugar (e.g. soft drinks, \\ nectar, etc.) \\ Lean milk \\ Whole milk \\ Filtered coffee \\ Other coffee \\ Tea \\ Sugar-rich beverages \\ Cakes, buns, waffles, sweet biscuits \\ Dessert \\ Chocolate, sweet candy \\ Chips \\ Sugar-rich spreads (e.g. honey, jam, peanut but- \\ ter, etc.)
}

NFBDG, Norwegian food-based dietary guidelines; NORDIET-FFQ, NORDIET food frequency questionnaire.

aSalted nuts not included. Upper limit of the range of acceptable intake is based on the proportion of energy (about $7 \%$ ) contribution of nuts to total energy intake: $30 \mathrm{~g}$ nuts contain about $180 \mathrm{kcal}$.

bIntake of at least $20 \mathrm{~g} / \mathrm{d}$ unsalted nuts if normal weight $(\mathrm{BMI}<25)$.

Intake of nuts between 20 and $30 \mathrm{~g} / \mathrm{d}$ if overweight (BMI $\geq 25)$.

'Lean milk with less than $1.5 \%$ fat.

eAcceptable amount of intake equal to one portion per week.

fDefined as dairy products (not cheese and milk) containing less than $20 \%$ fat and dairy products labelled as light or reduced fat or containing less than $950-\mathrm{I}, 150 \mathrm{kJb}$ energy.

:Defined as cheese containing less than 17\% fat, cheese labelled as light or reduced fat, or containing less than 950-I, I50 kJ energy.

hShould include at least half portion per day ( I portion = I glass of lean milk = $200 \mathrm{~g}$ ).

Whole milk with more than $3.5 \%$ fat.

iDefined as dairy products (not cheese and milk) containing more than 20\% fat and/or energy content more than $950-1, \mathrm{I} 50 \mathrm{~kJ}$.

kDefined as cheese containing more than 17\% fat, cheese not labelled as light/reduced fat, or containing more than $950-1$, I50 $\mathrm{kJ}$ energy.

'Acceptable amount of intake equal to one portion per week.

moderate intake defined as at least one portion of unprocessed meat per week.

${ }^{n}$ Acceptable amount of intake equal to one portion per week.

${ }^{\circ}$ Acceptable amount of intake equal to one small portion per week.

PAcceptable amount of intake equal to one large portion per week.

\section{Sample size}

A sample size of 40 men and 40 women allows the detection of differences of one portion of fruit or vegetable (one portion $=100 \mathrm{~g}$ ) between test and reference methods, assuming a standard deviation of 1.6 portion (or $160 \mathrm{~g}$ ) $(32,33)$ with a significance level of $5 \%$ and power of $80 \%$. Moreover, a sample size of 38 men and 38 women was needed to detect a correlation coefficient of 0.5 or higher, with a significance level of $5 \%$ and power of $90 \%$ (34).

\section{Statistical analysis}

Data were analysed using IBM SPSS Statistics, version 22. All $p$-values were two-sided with a significance level of $5 \%$. All data were checked for normal distribution by evaluating histograms, normal Q-Q plots, and the Kolmogorov-Smirnov test $(p>0.05)$.

All subject characteristics were normally distributed and are presented as means with 95\% confidence interval. The categorical data are presented as frequencies and 
percentages. Most of the estimates of food and beverage intakes were not normally distributed and therefore are presented as median, 5th, and 95th percentile. Depending on distribution, a Student's $t$-test or Mann-Whitney U test was used to compare two groups with regard to continuous variables. Categorical variables were compared by the Fischer exact test and Pearson chi-square test. Wilcoxon signed-rank test for paired data was used to check for difference in median intake between the two dietary methods (NORDIET-FFQ and WR).

Spearman's rank order correlation (rho) was calculated to explore the strength of the relationship between the continuous variables from the two different methods. We used the levels of agreement between two methods as defined by Hankin et al. (35), of which a correlation below 0.3 is poor, between 0.3 and 0.49 is fair, and above 0.5 is satisfactory. Kappa correlation was used to explore the strength of the relationship between the categorical variables of 'Oil, margarine, and butter' from the two different methods. Bland-Altman plots were used to explore bias such as over- or under-reporting (estimated by mean differences), limits of agreement (mean difference $\pm 1.96 \mathrm{SD})$, and presence of outliers in the data $(36,37)$.

To evaluate the participants' adherence to the dietary recommendations as described in Tables 1 and 2, we calculated the NORDIET-FFQs sensitivity and specificity compared with the WR. Sensitivity was defined as the percentage of subjects who reported not fulfilling the recommendations for both the NORDIET-FFQ and WR assessments divided by the number of patients not fulfilling the recommendations according to the WR only. Specificity was defined as the percentage of subjects who reported fulfilling the recommendations for both the NORDIET-FFQ and WR assessments divided by the number of subjects fulfilling the recommendations according to the WR only. Sensitivity and specificity above $60 \%$ was defined as good.

\section{Results}

Eighty-one participants accepted the invitation (92\% participation rate, Fig. 1). General characteristics of the study population are presented in Table 3 . Daily mean energy intakes estimated from the WR were 8.9 and $7.6 \mathrm{MJ}$ for men and women, respectively.

Intakes of food and beverages from the NORDIET-FFQ and WR Median intakes of food and beverages estimated from the NORDIET-FFQ and the WR are presented in Table 4. Overall, the NORDIET-FFQ was able to estimate intake of the main food groups in the Norwegian FBDG and that are associated with cancer risk, except for whole grain products, water, and red and processed meat. Correlation coefficients between intakes estimated from the NORDIET-FFQ and WR are presented in Table 5. Correlation coefficients ranged from 0.12 for unprocessed meat to 0.74 for

Table 3. Characteristics of the validation group, all participants in total and stratified by gender

\begin{tabular}{|c|c|c|c|c|}
\hline Variables & Total $(n=8 \mathrm{I})$ & Men $(n=44)$ & Women $(n=37)$ & $p$ \\
\hline \multicolumn{5}{|l|}{ Age, years } \\
\hline Mean $(95 \% \mathrm{Cl})$ & $65.0(63.4,66.6)$ & $65.4(63.1,67.7)$ & $64.5(62.1,66.9)$ & $0.59^{\mathrm{a}}$ \\
\hline Smokers, $n(\%)$ & $6(7.4)$ & $3(6.8)$ & $3(8.1)$ & $<1.0^{\mathrm{b}}$ \\
\hline Energy intake, $\mathrm{kJ}{ }^{\mathrm{d}}$ & $8,362(7,859,8,865)$ & $8,929(8,215,9,643)$ & $7,640(7,065,8,2 \mid 4)$ & $0.007^{a}$ \\
\hline \multicolumn{5}{|l|}{ Education, $n(\%)$} \\
\hline Primary school & $5(6.2)$ & $3(6.8)$ & $2(5.4)$ & $0.035^{\mathrm{b}}$ \\
\hline Lower secondary/high school & $35(43.2)$ & $22(50.0)$ & $13(3 \mid .5)$ & \\
\hline College/university & $4 \mid(50.6)$ & $19(43.2)$ & $22(59.5)$ & \\
\hline \multicolumn{5}{|l|}{ Anthropometry (mean, 95\% Cl) } \\
\hline Weight, kg & $78.26(74.5,82.1)$ & $85.7(81.9,89.4)$ & $70.0(64.7,75.3)$ & $<0.001^{\circ}$ \\
\hline Height, m & $1.73(1.7 \mathrm{I}, \mathrm{I} .75)$ & $1.78(1.76,1.80)$ & $1.67(1.65,1.68)$ & $<0.00 I^{2}$ \\
\hline $\mathrm{BMI}, \mathrm{kg} / \mathrm{m}^{2}$ & $26.1(25.0,27.2)$ & $26.9(25.8,28.2)$ & $25.1(23.4,26.9)$ & $0.73^{a}$ \\
\hline Waist circumference, $\mathrm{cm}$ & $93.8(90.6,97.1)$ & $99.9(96.9,102.3)$ & $87.0(82.2,91.9)$ & $<0.00 I^{\circ}$ \\
\hline Hip circumference, $\mathrm{cm}$ & $101.2(98.9,103.1)$ & $101.2(99.0,103.3)$ & $100.7(96.9,104.6)$ & $0.84^{\mathrm{a}}$ \\
\hline \multicolumn{5}{|c|}{ Tumour classification $n(\%)($ total $n=73$, men $n=38$, women $n=35$ ) } \\
\hline TNM I & $14(19.2)$ & $10(26.3)$ & $4(11.4)$ & 0.089 \\
\hline TNM II & $34(46.6)$ & $19(50.0)$ & $15(42.9)$ & \\
\hline TNM III & $25(34.2)$ & $9(23.7)$ & $16(45.7)$ & \\
\hline
\end{tabular}

TNM, tumour node metastases; BMI, body mass index.

aStudent's $t$-test.

${ }^{b}$ Fischer exact test (two-sided).

'Pearson's chi-square test.

dEstimated energy intake from the 7-day weighed food records. 


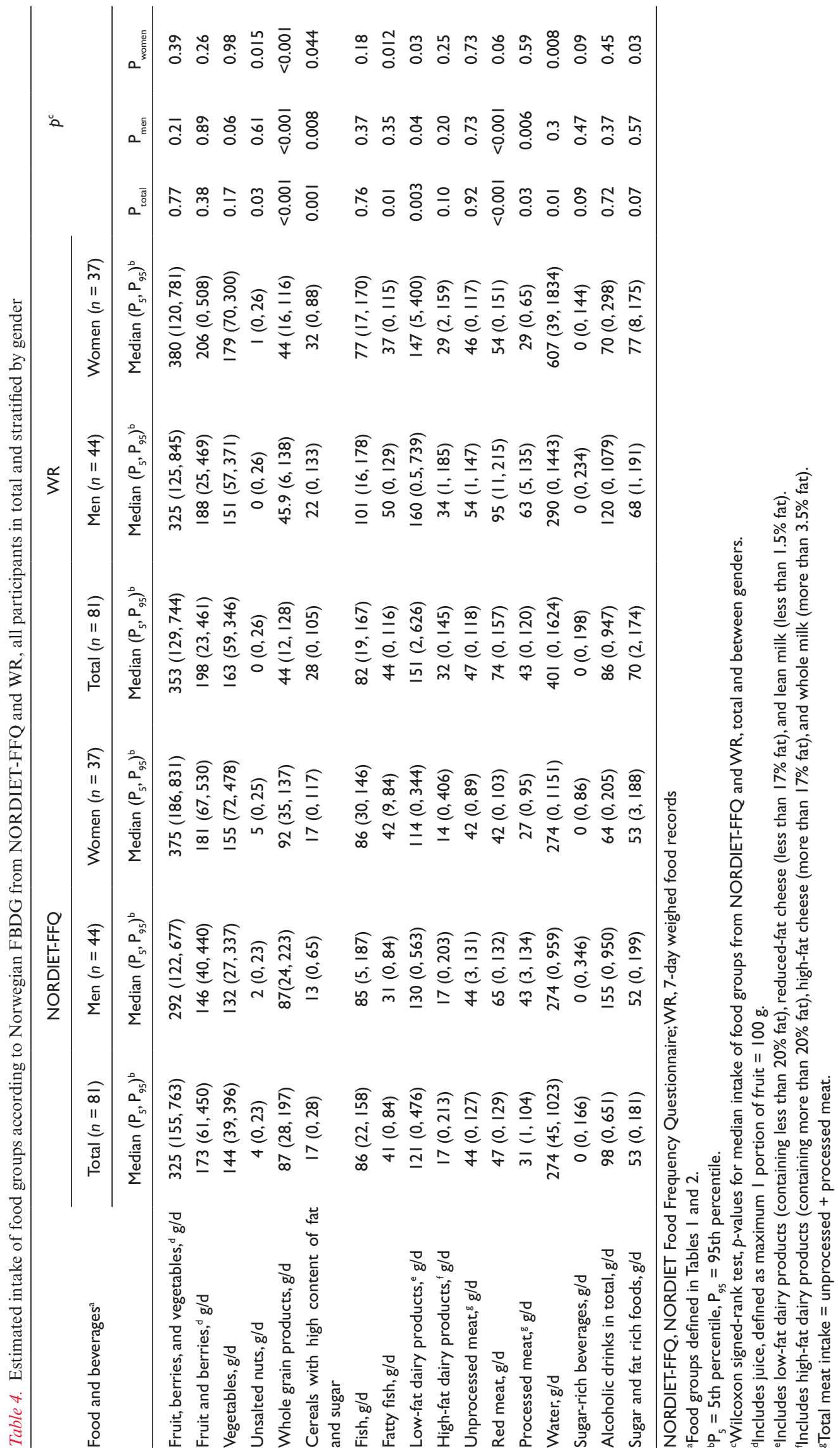


alcoholic beverages. However, most food groups showed fair and satisfactory correlations, with the exception of poor correlations for unprocessed meat, processed meat, and vegetables. Bland-Altman analyses are presented in Fig. 2 and Supplementary file 3. The majority of the plots (i.e. differences between methods on the $y$-axis against the mean value of methods on the $x$-axis) were within the $95 \%$ limit of agreement for each food group. At the upper level of intake of foods, there was a wider scatter of difference. Sensitivity and specificity analyses are presented in Table 6 . The NORDIET-FFQ was able to identify individuals in need of dietary counselling for most of the guidelines, with the exception of red meat, unprocessed meat, fish, and sugar-rich beverages. Estimated median intakes of the individual food items (ungrouped) are presented in Supplementary file 1 , with associated correlations coefficients between test and reference method in Supplementary file 2.

Fruits, berries, vegetables, and nuts

Median intakes of the food groups 'Fruits, berries, and vegetables', 'Fruits and berries', and 'Vegetables' did not differ significantly between the methods, for all participants in total or when divided by gender (Table 4). However, the limits of agreement were wide and under-reporting increased at high intakes (Fig. 2a and b, Supplementary file 3A). The Spearman's rho was fair for the first two food groups, but poor for 'Vegetables' (Table 5). The questions about fruits and vegetables in the NORDIET-FFQ showed sensitivity in the range of $84-87 \%$ and a low specificity ranging from 14 to $50 \%$ (Table 6).

The median intake of unsalted nuts did not differ significantly between the NORDIET-FFQ and WR for men only. Moreover, with regard to differences between the two methods the limits of agreements were $20 \mathrm{~g}$ above and below the mean difference (Fig. 2c). Spearman's rho was satisfactory (Table 5), the sensitivity was good, and specificity was low (Table 6).

\section{Whole grain products}

There was significant difference in median intakes of whole grain products and cereals with high content of fat and sugar between the two methods but with satisfactory Spearman's rho (Tables 4 and 5). The NORDIET-FFQ tended to report higher intakes in the category of 'Whole grain products' on the group level, with almost half of the recommended daily intake, which increased with higher intakes in both women and men (Fig. 2d). However, the NORDIET-FFQ was able to identify individuals not fulfilling the dietary recommendation for whole grain products among men and women, respectively (Table 6).

Table 5. Spearman rank order correlation $(r)$ of food and beverages groups between NORDIET-FFQ and WR, all participants in total and stratified by gender

\begin{tabular}{|c|c|c|c|}
\hline \multirow{2}{*}{ Foods and beverages ${ }^{a}$} & \multicolumn{3}{|c|}{ NORDIET-FFQ/WR, $r$} \\
\hline & Total $(n=8 \mathrm{I})$ & Men $(n=44)$ & Women $(n=37)$ \\
\hline Total fruit, berries, and vegetables ${ }^{\mathrm{b}}$ & $0.4 I^{*}$ & $0.42^{*}$ & 0.33 \\
\hline Fruit and berries ${ }^{\mathrm{b}}$ & $0.48 *$ & $0.49 *$ & $0.44^{*}$ \\
\hline Vegetables & 0.15 & 0.11 & 0.15 \\
\hline Unsalted nuts & $0.52 *$ & $0.58^{*}$ & 0.40 \\
\hline Whole grain products & $0.55^{*}$ & $0.68 *$ & 0.28 \\
\hline Cereals with high content of fat and sugar & $0.31 *$ & 0.23 & $0.40 *$ \\
\hline Fish & $0.37^{*}$ & $0.5 I^{*}$ & 0.18 \\
\hline Fatty fish & $0.35 *$ & $0.46 *$ & 0.14 \\
\hline Low-fat dairy products ${ }^{c}$ & $0.73 *$ & $0.78^{*}$ & $0.70 *$ \\
\hline High-fat dairy products ${ }^{d}$ & $0.46 *$ & 0.23 & $0.73 *$ \\
\hline Unprocessed meat & 0.12 & 0.11 & 0.15 \\
\hline Red meat & $0.45^{*}$ & $0.43 *$ & 0.39 \\
\hline Processed meat & 0.29 & 0.23 & 0.24 \\
\hline Water & $0.45^{*}$ & $0.40^{*}$ & $0.42^{*}$ \\
\hline Sugar-rich beverages & $0.46^{*}$ & $0.64^{*}$ & 0.16 \\
\hline Alcoholic drinks in total & $0.74 *$ & $0.78 *$ & $0.7 I *$ \\
\hline Sugar- and fat-rich foods & $0.49 *$ & $0.43 *$ & $0.61 *$ \\
\hline
\end{tabular}

NORDIET-FFQ, NORDIET food frequency questionnaire;WR, 7-day weighed food record

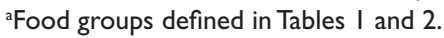

Includes juice, defined as maximum I portion of fruit $=100 \mathrm{~g}$.

Includes low-fat dairy products (containing less than $20 \%$ fat), reduced-fat (less than $17 \%$ fat) and lean milk (less than $1.5 \%$ fat).

Includes high-fat dairy products (containing more than $20 \%$ fat), high-fat cheese (more than $17 \%$ fat), and whole milk (more than $3.5 \%$ fat).

*Correlation is significant at the 0.01 level (two-tailed). 
(a)

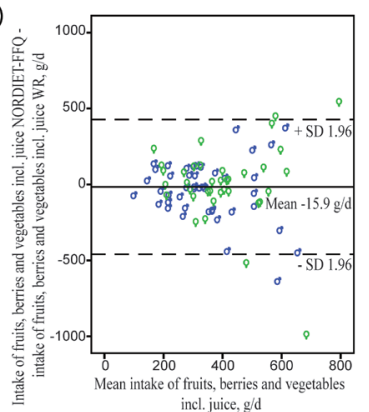

(d)

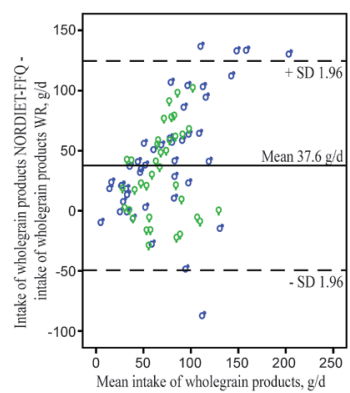

(g)

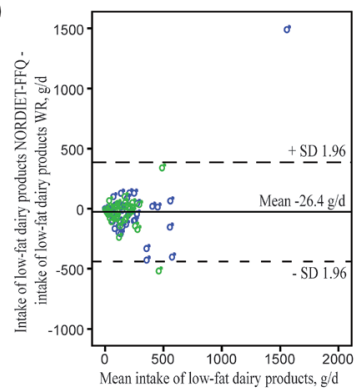

(j)

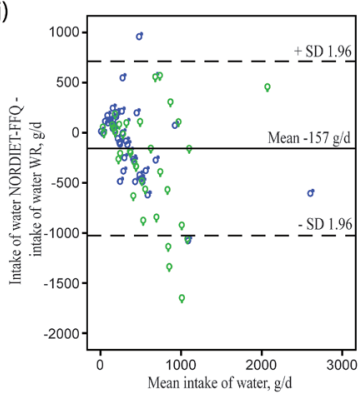

(b)

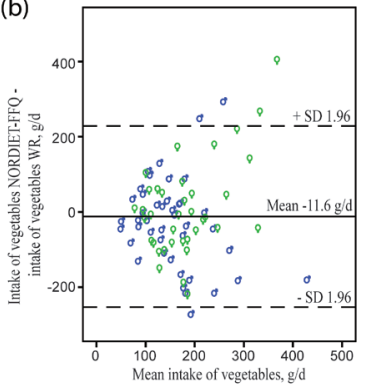

(e)

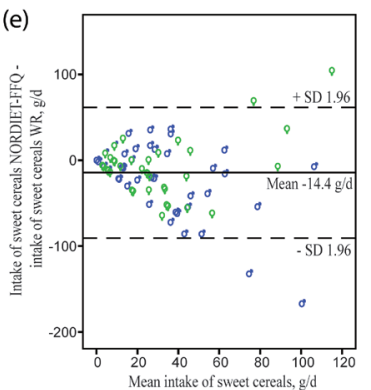

(h)

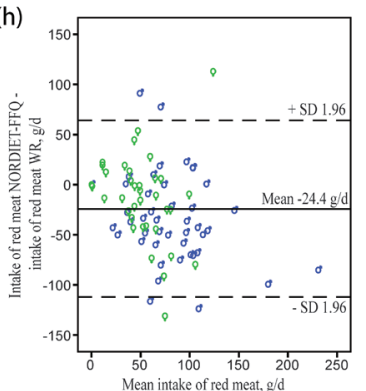

(k)

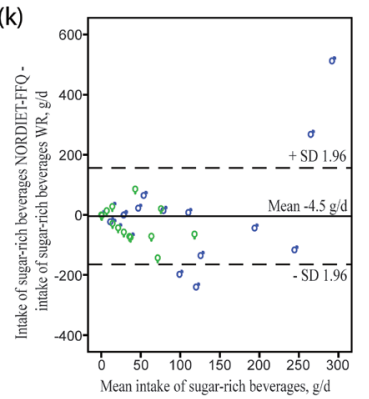

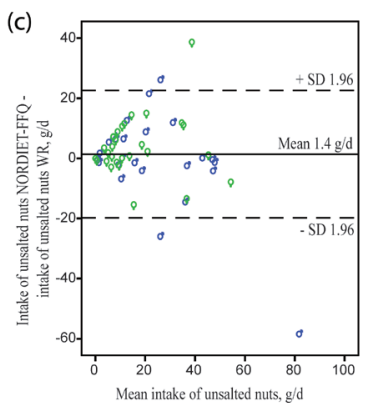

(f)

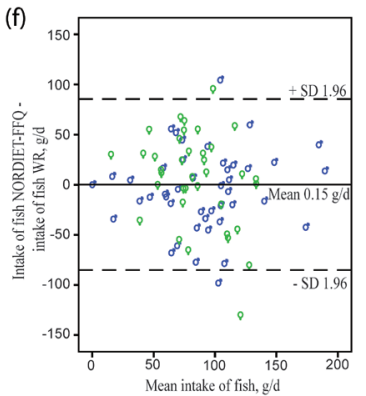

(i)

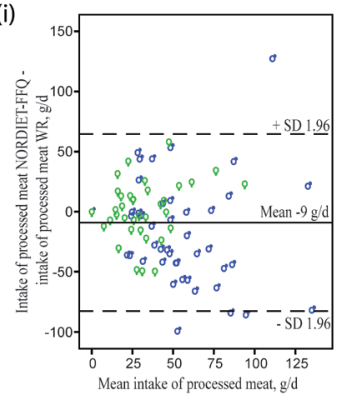

(I)

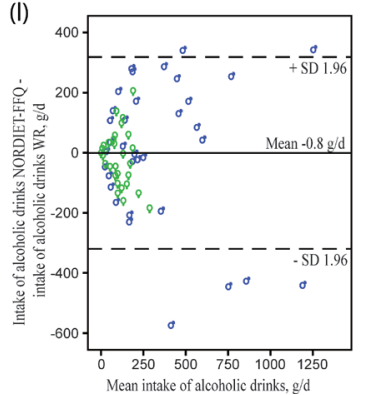

Fig. 2. Bland-Altman plots depicting the mean differences [NORDIET-FFQ minus weighed food dairy (WR)] for intake of food groups in grams per day: (a) fruits, berries, vegetables including juice; (b) vegetables; (c) unsalted nuts; (d) whole grain products; (e) sweet cereals; (f) fish; (g) low-fat dairy products; (h) red meat; (i) processed meat; (j) water; (k) sugar-rich beverages; (l) alcoholic drinks. The solid line represents the mean, and the dashed lines represent the 1.96 SDs of the observations. Females are denoted with the symbol $q$ the symboldwith the symbol $\hat{o}$.

The under-reporting of intake of the category 'Cereals with high content of fat and sugar' showed a trend towards increasing differences between methods with higher intakes (Fig. 2e). The questionnaire was only able to identify individuals fulfilling the recommendations of 'At least half of the grains should be whole grains' and 'Reduce cereals with a high content of fat, salt, and sugar' (Table 6).
Fish

Median intakes of fish did not differ significantly between the two assessment methods on group level. Estimated intakes of fatty fish were significantly different for women but not for men (Table 4). The differences in intakes of fish and fatty fish between the methods did not show any trend in the distribution but were scattered above and below the 
Table 6. Sensitivity and specificity of the NORDIET-FFQ to detect participants not complying or complying with the Norwegian FBDG relative to WR

\begin{tabular}{|c|c|c|}
\hline Guideline & Sensitivity $n^{\mathrm{a}}(\%)$ & Specificity $n^{\mathrm{b}}(\%)$ \\
\hline I.I Include vegetables, fruits, and berries in your daily diet. & $57(85.1)$ & $3(21.4)$ \\
\hline 1.2 About half of the intake should be as fruits and berries. & $48(84.2)$ & $12(50.0)$ \\
\hline 1.3 About half of the intake should be as vegetables. & $58(86.6)$ & $2(14.3)$ \\
\hline I.4 A moderate amount of unsalted nuts should be included in your daily diet. & $66(93.0)$ & $5(55.6)$ \\
\hline \multicolumn{3}{|l|}{ 2.I: } \\
\hline - Men should include at least $90 \mathrm{~g}$ of whole grains in their daily diet. & $21(66.7)$ & $4(55.3)$ \\
\hline - Women should include at least $70 \mathrm{~g}$ of whole grains in their daily diet. & $13(100)$ & $7(43.3)$ \\
\hline 2.2 At least half of the grains should be whole grains. & $5(29.4)$ & $62(96.9)$ \\
\hline 2.3 Reduce cereals with high content of fat, salt, and sugar. & $20(41.7)$ & $27(81.8)$ \\
\hline 3.I Include intake of fish in your diet. & $5(41.7)$ & $64(92.8)$ \\
\hline 3.2 Include fatty fish in your diet. & $9(34.6)$ & $45(81.8)$ \\
\hline 4.I Low-fat dairy products should be included in your daily diet. & $21(80.8)$ & $39(70.9)$ \\
\hline 4.2 High-fat dairy products should be limited. & $34(57.6)$ & $17(77.3)$ \\
\hline 5.I Include unprocessed meat in your usual diet. & I (8.0) & $61(89.7)$ \\
\hline 5.2 Limit intake of red meat (beef, pork, lamb, goat). & $16(38.1)$ & $32(82.1)$ \\
\hline 5.3 Give preference to unprocessed meat over processed meat. & $12(38.7)$ & $33(66.0)$ \\
\hline 5.4 Reduce intake of processed meat. & $5 \mathrm{I}(82.3)$ & $7(36.8)$ \\
\hline $\begin{array}{l}\text { 6.I It is recommended to use cooking oil, liquid margarine, or soft margarine more } \\
\text { than butter with a high content of saturated fatty acids. }\end{array}$ & $15(46.9)$ & $40(81.6)$ \\
\hline 7.I Water is recommended as the primary choice of drinks. & $25(73.5)$ & $23(48.9)$ \\
\hline 7.2 Reduce sugar-rich beverages. & $14(5 \mid .9)$ & $48(88.9)$ \\
\hline 7.3 No alcohol intake. & $57(96.6)$ & $16(72.7)$ \\
\hline 8.I Reduce intake of foods with high content of sugar and fat. & $63(92.6)$ & $8(6 \mid .5)$ \\
\hline
\end{tabular}

Norwegian FBDG, Norwegian food-based dietary guidelines, NORDIET-FFQ, NORDIET food frequency questionnaire;WR, 7-day weighed food record. aSubjects reported not fulfilling the recommendations for both the NORDIET-FFQ and WR.

bSubjects reported fulfilling the recommendations for both the NORDIET-FFQ and WR.

mean differences (Fig. 2f and Supplementary file 3B). The Spearman's rho was fair, sensitivity was low, and the specificity was high for both food groups (Tables 4 through 6).

\section{Dairy products}

The median intake of low-fat dairy products was significantly different between the two methods, but the Spearman's rho was satisfactory (Tables 4 and 5). The differences between the methods were evenly distributed above and below the mean difference, which showed a mean under-reporting of $26 \mathrm{~g} / \mathrm{d}$ on the group level. The limits of agreement were mostly within the amount of two glasses of milk (Fig. 2). The median intake of high fat dairy products was not significantly different between the methods, and the Spearman's rho was fair for the total population, poor for men, and satisfactory for women (Tables 4 and 5). Both sensitivity and specificity were high for low fat dairy products, but the sensitivity was lower for high fat dairy products (Table 6).

\section{Meat}

The NORDIET-FFQ was able to estimate intakes of unprocessed meat, red meat (only women), and processed meat (only women) on the group level (Table 4). Moreover, red meat showed a fair Spearman's rho, whereas unprocessed meat and processed meat showed a poor Spearman's rho (Table 4). The limits of agreements were almost within $140 \mathrm{~g}$ (Fig. 2h and i, Supplementary file 3D). The NORDIET-FFQ was only able to identify individuals in need of dietary counselling for intakes of processed meat (Table 6). However, the NORDIET-FFQ was able to identify those who followed the recommendations for intakes of red meat and unprocessed meat (specificity, 90 and $82 \%$ ) but not for processed meat (specificity of $37 \%$ ) (Table 6).

\section{Oil, margarine, and butter}

The NORDIET-FFQ was able to identify participants who fulfilled the recommendations for intakes of dietary fat in their diets but not those who did not fulfil the recommendations (Table 6). Moreover, the measure of agreement between the methods was poor (kappa coefficient $=$ $0.29, p<0.006$ ).

\section{Water and other beverages}

Median intakes of water were significantly different for women and the total population and the Spearman's rho was fair (Tables 4 and 5). The underestimation of water from the NORDIET-FFQ increased with higher intakes 
(Fig. 2j). Median intakes of beverages with added sugar were not significantly different between the methods and the Spearman's rho was fair for all participants in total, and satisfactory and poor among men and women, respectively (Tables 4 and 5, Fig. 2k). There were no significant difference between the methods in median intakes of alcoholic drinks in total and the Spearman's rho was satisfactory (Tables 4 and 5). The sensitivity analyses of beverages ranged from $52 \%$ ('Reduce sugar rich beverages') to $97 \%$ ('No alcoholic intake'), and the specificity ranged from $49 \%$ ('Water is recommended as the primary choice of drink') to $89 \%$ ('Reduce sugar-rich beverages') (Table 6).

\section{Sugar- and fat-rich foods}

The NORDIET-FFQ was able to estimate median intakes and to rank individual intakes of sugar- and fat-rich foods on the group level (Tables 4 and 5, Supplementary file 3E). The NORDIET-FFQ was also able to identify those not fulfilling the recommendation and moderately those who fulfilled the recommendation (Table 6).

\section{Discussion}

In the present study, we evaluated the NORDIET-FFQ's ability to assess adherence to the Norwegian FBDG in CRC patients. The NORDIET-FFQ was able to estimate intakes of the main dietary guidelines, such as fruits, vegetables, fish, meat, high-fat dairy products, beverages, and energy-dense foods. Only three food groups showed significant differences between methods for both genders, of which 'Low-fat dairy products' and 'Cereals with high content of sugar and fat' were under-reported and the 'Whole grain products' was over-reported in the NORDIET-FFQ compared to the WR. We speculate that the under-reporting of unhealthy cereals and the over-reporting of healthy whole grain products may be a result of social desirability bias, as seen in other studies, where presumed unhealthy foods are under-reported and healthy foods are over-reported (38-40). The underreporting of 'Low fat dairy products' may also be explained by participants having difficulty interpreting the dairy questions and not knowing if the dairy products they use are high- or lowfat products. There is a wide range of dairy products on the market with varying fat content and a valid intake estimate of this food category relies heavily on participants' knowledge of fat content in the products they consume. Thus, this may reduce the ability of the NORDIET-FFQ to estimate intakes of low-fat products. The estimate of the category 'Low fat dairy products' was an aggregation of the following three entries: 'Low-fat milk', 'Cheese with low fat content', and 'Dairy products with low-fat content'. Each of these entries gives valid estimates of intakes. Thus, the single questions regarding dairy foods gave better estimates when used separately.
Moreover, stratifying by gender increased the estimation of intakes for additional food groups. Gender differences in dietary assessment methods have been reported in other studies as well $(8,14,41)$. Lee and co-workers (41) emphasise the importance of including different portion sizes for men and women in FFQs, due to their findings that when gender is not considered, greater inaccuracy in dietary intake assessment is found in women compared to in men. In the present study, portions sizes were equal for men and women and they reported intakes of nuts, fish, water, meat, and sugar- and fat-rich foods differently on the NORDIET-FFQ.

Short FFQs have been shown to be able to classify individuals according to intakes of food groups and nutrients $(12,15,20,23,36,37)$. In the present study, the NORDIET-FFQ showed fair to satisfactory agreement with respect to ranking individuals by their dietary intake compared to WR. The Spearman's rho ranged from 0.12 to 0.74 , with most correlations categorised as fair or satisfactory and statistically significant $(p<0.01)$ (Tables 5 and Supplementary file 2). This is consistent with other similar studies showing a correlation coefficient for foods and nutrients ranging from 0.3 to $0.7(13,15,17,42)$. Carlsen and co-workers found that correlations between intakes of fruit and vegetable from a long FFQ and WR ranged from 0.31 to 0.58 (43). Importantly, in the present study, a fair or satisfactory correlation was documented for the foods that are shown to be associated with risk of CRC, such as fruits and vegetables, whole grains, dairy products, red and processed meat, and alcoholic beverages $(2-4,44)$. However, three food groups showed poor correlations for both genders and in total, that is vegetables, unprocessed meat, and processed meat. Classifying individuals according to intake of food groups may be advantageous in clinical intervention studies as a measure of intervention effects, as well as in a clinical setting to identify patients 'at risk' (23). Based on the high number of fair and satisfactory correlations observed in this study the NORDIET-FFQ's ability to rank participants according to food intake is fair and comparable to other short FFQs $(11,17,20,26,45-48)$.

Correlation coefficients measures associations between a questionnaire and its reference method but are unable to detect systematic errors that may be of clinical importance (37).

When assessing the agreements between two methods, Bland-Altman plots are recommended $(34,36,37)$. Overall, the Bland-Altman plots in the present study showed wide limits of agreement for most of the food groups and a trend towards increased over- or under-reporting with higher mean intakes. However, the mean differences were smaller than $20 \%$ between the methods for most of the food groups and the limits of agreement were almost within a daily portion for fruits, vegetables, nuts, fish, meat, and dairy products. Systematic and random errors 
can more easily be revealed by Bland-Altman plots, as the data points are less compressed compared to a scatter plot. For instance, in the present study 'Whole grain products' showed a satisfactory correlation, but a systematic error was revealed by the Bland-Altman plot, showing increasing over-reporting with increasing intakes. Moreover, 'Fish' showed a fair correlation and the distribution of differences against the mean value of the two methods did not show any clear trend. Intakes in the category of 'Vegetables' showed poor correlation and an increased under-reporting with higher intakes, indicating poor ability of the NORDIET-FFQ to measure intakes of vegetables. This may be due to the difficulty of reporting portions of vegetables, since these foods are often included in dishes, compared to fruits, which are more often eaten raw in one unit (43). However, the questionnaire was good in measuring intakes of vegetables on the group level and according to the recommendations. Thus the NORDIET-FFQ gave a fairly good estimate of intake for several of the food groups on the group level compared to the WR standard.

The NORDIET-FFQ was able to detect individuals not fulfilling the Norwegian FBDG (i.e. sensitivity) for 10 out of 20 recommendations, covering most of the food groups shown to be associated with CRC risk (i.e. fruits and vegetables, whole grains, processed meat, alcohol intake, dairy products) $(1-4,44)$. Moreover, the NORDIET-FFQ was able to identify individuals who fulfilled 13 out of 20 recommendations (i.e. specificity) (Table 6).

\section{Strengths and limitations}

Attenuation of agreement between two methods can occur due to different time period covered by the methods. In the present study we compared a retrospective method (NORDIET-FFQ) with a prospective method (WR). A limitation of the study may be that the NORDIET-FFQ asked for dietary intake over the previous 1-2 months, whereas the WR recorded dietary intakes for the subsequent week. However, the short time period covered by this study design, approximately 2.5 months, would probably limit the error between NORDIET-FFQ and WR recording $(8,14)$. Timing and sequence for the test and reference method is important in validation studies, of which the test method should be administrated prior to the reference method (14). In the present study, the participants completed the FFQ prior to the WR and thereby avoided any learning effects from the reference method. Seasonal effects of dietary intakes were not expected since dietary intakes were collected from all seasons during a year. Moreover, variation in reporting of dietary intakes may be attributed to the comparison between closed and open ended methods (14). Study participants were recruited from both arms of the intervention. The purpose of an evaluation study is to compare estimates of intake of test and reference method. Hence, the diet of the participants should be assessed alike and should not be dependent on differences in diet among the participants. Both groups were equally exposed to the dietary assessment methods, anthropometric measurements, and biological samplings, as well as the direct contact with the researchers. However, we cannot rule out that the intervention group might have been more conscious about the registration of food and beverages than the participants in the non-intervention group.

FFQs are associated with limitations such as fixed food list, memory, and perception of portion sizes (8). However, WRs control these errors due to their independency of memory and direct measurement of food intakes (8, 16). In order to be used as a standard reference method in assessing the validity of questionnaires, WRs should cover a sufficient number of days to represent average dietary intakes (8). In the present study this was taken into account, since all the WRs recorded dietary intakes over 7 consecutive days.

As the aim of the NORDIET-FFQ was to measure adherence to the dietary guidelines, an aggregation of the single foods was needed. Hence, using the aggregated questions conforming to the Norwegian FBDG in the NORDIET-FFQ resulted in less detailed information of food intake. However, short FFQs containing aggregated questions have been shown to capture nearly as much information on dietary intakes as long FFQs $(8,16)$.

\section{Conclusion}

The NORDIET-FFQ was able, on a group level, to estimate intakes of most food groups related to the Norwegian FBDG, such as fruits, vegetables, nuts, fish, dairy products, meat, beverages, and sugar- and fat-rich foods. Moreover, the NORDIET-FFQ was able to rank individual intakes and to identify those individuals in need of dietary counselling for foods that are shown to be associated with risk of $\mathrm{CRC}$, such as fruit and vegetables, whole grains, red meat, alcoholic beverages, and dairy products. The NORDIET-FFQ was not able to rank individual intakes of processed meat, unprocessed meat, and vegetables. Overall, the NORDIET-FFQ gives valid estimates of dietary intake according to the Norwegian FBDG.

\section{Acknowledgements}

We would like to thank the patients for their valuable contribution to this study. We would also like to thank Stine Fallingen Ødegaard, Hanna Ræder, Siv Åshild Billington, Torgrim Langleite, Mari Bøe Sebelien, Magnhild Håskjold, Jonas Grenne, and Karoline Borg for their contributions to data collection and study administration.

\section{Conflict of interest and funding}

All other authors declare that they have no competing interests. This project has received funding from the Research Council of Norway, Throne Holst Foundation of Nutrition Research, Norwegian Cancer Society, and South Eastern Norway Regional Health Authority. 


\section{Authors' contributions}

$\mathrm{HBH}$ had primary responsibility for writing the manuscript. $\mathrm{HBH}$, MHC, IP, SKB, AJS, ASK, LFA, CH, SS, SB, and RB contributed to the conception and design of the study, analysis and interpretation of the data, and drafting of the manuscript. HBH, AJS, IP, and ASK contributed to acquisition of data. All authors contributed to the writing and approval of the final manuscript.

\section{References}

1. Diet, nutrition and the prevention of chronic diseases: report of Joint WHO/FAO expert consultation on Diet, Nutrition and the Prevention of Chronic Diseases (2002, Geneva, Switzerland). Diet, Nutrition and the Prevention of Chronic Diseases: report of a joint WHO/FAO expert consultation, Geneva, 28 January 1 February 2002.

2. World Cancer Research Fund/American Institute for Cancer Research (2007). Food, nutrition, physical activity, and the prevention of cancer: a global perspective. Washington, DC: American Institute for Cancer Research.

3. Kostråd for å fremme folkehelsen og forebygge kroniske sykdommer: metodologi og vitenskapelig kunnskapsgrunnlag. Oslo: Nasjonalt råd for ernæring, Helsedirektoratet; 2011. Directorate of Health Oslo, Norway.

4. Diet, nutrition, physical activity and colorectal cancer. Continuous Update Project Report World Cancer Research Fund/American Institute for Cancer Research. 2017.

5. Food and Agriculture Organization of the United Nations (FAO). Food-based dietary guidelines. Available from: http:// www.fao.org/nutrition/education/food-dietary-guidelines/home/ en/ [cited 1 January 2016].

6. Cancer in Norway 2016 (2017). Cancer incidence, mortality and prevalence in Norway. Oslo: Cancer registry of Norway.

7. Henriksen HB, Ræder H, Bohn SK, Paur I, Kværner AS, Billington SÅW, et al. .The Norwegian dietary guidelines and colorectal cancer survival (CRC-NORDIET) study: a food-based multicenter randomized controlled trial. BMC Cancer 2017; 17: 83.

8. Willett W. Nutritional epidemiology. Oxford: Oxford University Press; 2013.

9. Jia X, Craig LC, Aucott LS, Milne AC, McNeill G. Repeatability and validity of a food frequency questionnaire in free-living older people in relation to cognitive function. J Nutr Health Aging 2008; 12: 735-41.

10. Dumartheray EW, Krieg MA, Cornuz J, Whittamore DR, Lovell DP, Burckhardt P, et al. Validation and reproducibility of a semi-quantitative Food Frequency Questionnaire for use in elderly Swiss women. J Hum Nutr Diet 2006; 19: 321-30.

11. Eysteinsdottir T, Thorsdottir I, Gunnarsdottir I, Steingrimsdottir L. Assessing validity of a short food frequency questionnaire on present dietary intake of elderly Icelanders. Nutr J 2012; 11: 12.

12. Kristjansdottir AG, Andersen LF, Haraldsdottir J, de Almeida $\mathrm{MD}$, Thorsdottir I. Validity of a questionnaire to assess fruit and vegetable intake in adults. Eur J Clin Nutr 2006; 60: 408-15.

13. Lillegaard IT, Overby NC, Andersen LF. Evaluation of a short food frequency questionnaire used among Norwegian children. Food Nutr Res 2012; 56. 6399 - DOI:10.3402/fnr.v56i0.6399

14. Margetts BM, Nelson,M. Design concepts in nutritional epidemiology. New York: Oxford University Press; 1997.

15. Thompson FE, Byers T. Dietary assessment resource manual. J Nutr 1994; 124: 2245s-317s.

16. Willett WC, Sampson L, Stampfer MJ, Rosner B, Bain C, Witschi $\mathrm{J}$, et al. Reproducibility and validity of a semiquantitative food frequency questionnaire. Am J Epidemiol 1985; 122: 51-65.
17. Yokoyama Y, Takachi R, Ishihara J, Ishii Y, Sasazuki S, Sawada $\mathrm{N}$, et al. Validity of short and long self-administered food frequency questionnaires in ranking dietary intake in middle-aged and elderly Japanese in the Japan Public Health Center - based prospective study for the next generation (JPHC-NEXT) protocol area. J Epidemiol 2016; 26: 12.

18. Osler M, Heitmann BL. The validity of a short food frequency questionnaire and its ability to measure changes in food intake: a longitudinal study. Int J Epidemiol 1996; 25: 1023-9.

19. Xinying PX, Noakes M, Keogh J. Can a food frequency questionnaire be used to capture dietary intake data in a 4 week clinical intervention trial? Asia Pac J Clin Nutr 2004; 13: 318-23.

20. England CY, Andrews RC, Jago R, Thompson JL. A systematic review of brief dietary questionnaires suitable for clinical use in the prevention and management of obesity, cardiovascular disease and type 2 diabetes. Eur J Clin Nutr 2015; 69: 977-1003.

21. Asaad G, Sadegian M, Lau R, Xu Y, Soria-Contreras DC, Bell $\mathrm{RC}$, et al. The reliability and validity of the perceived dietary adherence questionnaire for people with type 2 diabetes. Nutrients 2015; 7: 5484-96.

22. Bell LK, Golley RK, Magarey AM. A short food-group-based dietary questionnaire is reliable and valid for assessing toddlers' dietary risk in relatively advantaged samples. Br J Nutr 2014; 112: 627-37.

23. Cade J, Thompson R, Burley V, Warm D. Development, validation and utilisation of food-frequency questionnaires - a review. Public Health Nutr 2002; 5: 567-87.

24. Golley RK, Bell LK, Hendrie GA, Rangan AM, Spence A, McNaughton SA, et al. Validity of short food questionnaire items to measure intake in children and adolescents: a systematic review. J Hum Nutr Diet 2017; 30: 36-50.

25. Kupek E, de Assis MA, Bellisle F, Lobo AS. Validity of WebCAAFE questionnaire for assessment of schoolchildren's dietary compliance with Brazilian Food Guidelines. Public Health Nutr 2016; 19: 2347-56.

26. Perez Rodrigo C, Moran Fagundez LJ, Riobo Servan P, Aranceta Bartrina J. Screeners and brief assessment methods. Nutr Hosp 2015; 31(Suppl 3): 91-8.

27. Randall Simpson J, Gumbley J, Whyte K, Lac J, Morra C, Rysdale L, et al. Development, reliability, and validity testing of Toddler NutriSTEP: a nutrition risk screening questionnaire for children 18-35 months of age. Appl Physiol Nutr Metab 2015; 40: 877-86.

28. Edge SB. AJCC cancer staging handbook: from the AJCC cancer staging manual. New York: Springer; 2009.

29. Rimestad AL, Løken EB, Nordbotten A. The Norwegian food composition table and the database for nutrient calculations at the Institute for Nutrition Research. Norwegian J Epidemiol 2000; $10: 16$.

30. The Keyhole symbole Oslo, Norway. Available from: http:// www.nokkelhullsmerket.no/ [cited 1 December 2015].

31. Brødskalaen Norwegian Directorate of Health Oslo, Norway. Available from: http://www.matportalen.no/merking/tema/ merking_av_mat/bruk_brodskalan [cited 1 January 2016].

32. Glasson C, Chapman K, James E. Fruit and vegetables should be targeted separately in health promotion programmes: differences in consumption levels, barriers, knowledge and stages of readiness for change. Public Health Nutr 2011; 14: 694-701.

33. Totland TH. Norkost 3: en landsomfattende kostholdsundersøkelse blant menn og kvinner i Norge i alderen 18-70 år, 2010-11. Oslo: Helsedirektoratet; 2012.

34. Bland M. An introduction to medical statistics. 3rd ed. Oxford: Oxford University Press; 2000. 
35. Hankin JH, Wilkens LR, Kolonel LN, Yoshizawa CN. Validation of a quantitative diet history method in Hawaii. Am J Epidemiol 1991; 133: 616-28.

36. Bland JM, Altman DG. Statistical methods for assessing agreement between two methods of clinical measurement. Lancet 1986; 1: 307-10.

37. Schmidt ME, Steindorf K. Statistical methods for the validation of questionnaires - discrepancy between theory and practice. Methods Inf Med 2006; 45: 409-13.

38. Kipnis V, Midthune D, Freedman LS, Bingham S, Schatzkin A, Subar A, et al. Empirical evidence of correlated biases in dietary assessment instruments and its implications. Am J Epidemiol 2001; 153: 394-403.

39. Tooze JA, Freedman LS, Carroll RJ, Midthune D, Kipnis V. The impact of stratification by implausible energy reporting status on estimates of diet-health relationships. Biom J 2016; 58: 1538-51.

40. Willett WC. Diet and cancer: an evolving picture. JAMA 2005; 293: 233-4.

41. Lee H, Kang M, Song WO, Shim JE, Paik HY. Gender analysis in the development and validation of FFQ: a systematic review. Br J Nutr. 2016;115:666-71.

42. Carlsen MH, Lillegaard IT, Karlsen A, Blomhoff R, Drevon CA, Andersen LF. Evaluation of energy and dietary intake estimates from a food frequency questionnaire using independent energy expenditure measurement and weighed food records. Nutr J 2010; 9: 37.

43. Carlsen MH, Karlsen A, Lillegaard IT, Gran JM, Drevon CA, Blomhoff $\mathrm{R}$, et al. Relative validity of fruit and vegetable intake estimated from an FFQ, using carotenoid and flavonoid biomarkers and the method of triads. Br J Nutr 2011; 105: $1530-8$.

44. Continuous Update Project Report (2011). Food, nutrition, physical activity, and the prevention of colorectal cancer. Washington, DC: World Cancer Research Fund/American Institute for Cancer Research.

45. Andersen LF, Johansson L, Solvoll K. Usefulness of a short food frequency questionnaire for screening of low intake of fruit and vegetable and for intake of fat. Eur J Public Health 2002; 12: 208-13.

46. Saeedi P, Skeaff SA, Wong JE, Skidmore PM. Reproducibility and relative validity of a short food frequency questionnaire in 9-10 year-old children. Nutrients 2016; 8. 271 - DOI:10.3390/ nu8050271

47. Saloheimo T, Gonzalez SA, Erkkola M, Milauskas DM, Meisel $\mathrm{JD}$, Champagne CM, et al. The reliability and validity of a short food frequency questionnaire among 9-11-year olds: a multinational study on three middle-income and high-income countries. Int J Obes Suppl 2015; 5: S22-8.

48. Schroder H, Benitez Arciniega A, Soler C, Covas MI, Baena-Diez $\mathrm{JM}$, Marrugat J. Validity of two short screeners for diet quality in time-limited settings. Public Health Nutr 2012; 15: 618-26.

\section{*Rune Blomhoff}

Department of Nutrition, Institute of Basic Medical Sciences, University of Oslo, Oslo, Norway, P.O.Box 1049, Blindern, NO-03 16 Oslo, Norway

Email: rune.blomhoff@medisin.uio.no 